\title{
Renegotiated CBR Transmission in Interactive Video-on-Demand System
}

\author{
Noriaki Kamiyama, Victor O.K. Li \\ Communication Sciences Institute \\ University of Southern California \\ Los Angeles, CA 90089-2565 \\ Email:\{noriaki,vli\}@irving.usc.edu
}

\begin{abstract}
An Interactive Video-On-Demand (IVOD) system requires transmission bandwidth allocation for each user. Since the volume of data in each video frame is variable, dynamic bandwidth allocation is desirable. In this paper, a new scheme that dynamically determines required bandwidth based on the queue length at the viewers Set-Top Box (STB) is proposed. This method requires no precalculation, so it is easily applied to IVOD. The variance of the video transmission rate for each user is an important factor as it affects the service quality of other multiplexed traffic. It is desirable that the transmission rate is changed gradually. A multi-layer concept is introduced to achieve this. Through numerical evaluation using actual movie data, we demonstrate that the variance of the transmission rate is close to the optimal value and the bandwidth utilization is close to unity.
\end{abstract}

\section{Introduction}

A Video-On-Demand (VOD) system enables a viewer to choose a video program from a large selection without leaving his home. It can offer a wide range of services. One extreme is Near VOD (NVOD) service in which a pointto-multipoint stream is generated and multiple viewers are serviced by one stream to reduce the system cost at the expense of service delay (the viewer must request playback between five and 30 minutes prior to the start of playback[1]). The other extreme is Interactive VOD (IVOD) services in which a stream is provided to each individual viewer with full interactivity. Since the viewer can use the stream privately, the video begins to play upon request without delay. VCR-like controls, i.e. pause, fast forward, rewind, etc. are also available.

The VOD system consists of a video server, a backbone network, an access network, and a Set-Top Box (STB)[2]. The video server stores encoded video and retrieves it upon request. The retrieved data is transmitted over the backbone and access network to the STB in the viewer's home. The main functions of STB are to absorb delay jitter and to decode video frames. Video is encoded into digital data and stored in the video server. The encoder can be classified into two categories: variable rate and constant rate. The variable rate encoder provides constant video quality, while the constant rate encoder gives variable quality services. Although variable quality services can be provided at a lower cost, the quality degradation is severe in frames whose redundancy are small (such as a scene change). Therefore, constant quality service is preferable. A popular encoding scheme, MPEG, belongs to this category[3]. In this paper, we only consider the IVOD system which provides constant quality service.

Since most existing networks provide constant bit rate (CBR) service, the accommodation of variable bit rate (VBR) video on $\mathrm{CBR}$ network has received much attention in the literature. The challenge is due to the bursty nature of VBR video and its delay sensitivity. The delay sensitivity makes it difficult to apply closed-loop congestion control schemes. So open-loop congestion control is necessary for VBR video service. However, this control is also difficult because of the bursty nature of VBR video. To allocate bandwidth, the traffic parameters must be specified for Call Admission Control (CAC). Moreover, specified traffic parameters must be enforced at each Network User Interface (NUI) in a packetswitched network (this control is called Usage Parameter Control (UPC)). This traffic specification is quite difficult for VBR video. Moreover, it seems difficult to obtain a statistical multiplexing gain by multiplexing many VBR video sources because they have a self-similar property[4].

Since CAC and UPC are simple for CBR traffic, one approach [5],[6] is to treat VBR video as CBR. Although this resolves the above problems, large pre-loading delay ${ }^{\dagger}$ and memory size are required to fit VBR traffic with a constant allocated bandwidth. Another solution is to renegotiate the CBR rate during the session, thereby decreasing the required pre-loading delay and STB memory[7],[8],,[9]. However, the complex pre-calculation and associated delay necessary to obtain a bandwidth sequence table makes the scheme unsuitable for a real-time application such as IVOD. Whenever a viewer makes an interactive operation, he must wait for the pre-calculation.

In this paper, we propose a new dynamic bandwidth allocation method. Although this method is in the renegotiated CBR category, it needs no pre-calculation. Therefore, IVOD with constant quality service is easily realized. To avoid both overflow and underflow at STB, the allocated bandwidth is determined based on the queue length at STB. The Coefficient of Variation (CoV) of the transmission rate (allocated bandwidth) should be as small as possible to minimize the impact on other traffic. Hence, we introduce a multiple layer approach to deal with the fluctuation of the STB queue length. Using real data from the MPEG encoded movie Star Wars, it is demonstrated that

${ }^{\dagger}$ The pre-loading delay is the time difference between the arrival of the first data frame at the STB and the beginning of the playback. This delay is introduced to avoid STB buffer underflow. 
the $\mathrm{CoV}$ of the transmission rate is close to the ideal value and the network utilization is close to unity.

The remainder of this paper is organized as follows. In Section 2, we review previously proposed bandwidth allocation methods. In Section 3, we define the proposed scheme. We introduce the multi-layer approach in Section 4. In Section 5 , we investigate the performance of the new method using actual MPEG traces. Finally, in Section 6, we conclude our study.

\section{Bandwidth Allocation in VOD}

In this section, existing bandwidth allocation methods proposed for stored video transmission is briefly summarized.

Video playback requires continuous data arrival. If the STB buffer is empty at a new frame playback time, the video playback is interrupted. New arriving data is discarded when the STB buffer is full; this degrades the quality. Therefore, a bandwidth allocation method is required to avoid both buffer underflow and overflow at STB.

CBR and Renegotiated CBR (RCBR)[5],[6],[8] bandwidth allocation methods can be classified into two major categories: (1) fixed allocation (CBR) and (2) dynamic allocation (RCBR). Moreover, these methods are distinguished by the frame period treatment in the network (see Fig. 1). One maintains a frame period, and the other transmits video data without considering a frame period.

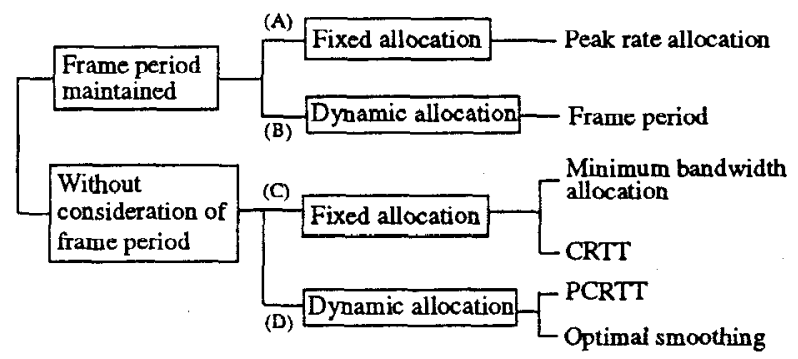

Figure 1: CBR accommodation methods on VOD

In the former, the video data forming one frame arrives at STB during one frame period, so STB buffer equal to one maximum frame size is enough to avoid both overflow and underflow. If the CBR bandwidth is allocated only at the call set-up time ( $(A)$ in Fig. 1), the peak rate must be used. Although this allocation is the simplest, network utilization is low. Dynamic bandwidth allocation with frame period preservation ((B) in Fig. 1) can be also considered. This requires bandwidth renegotiation at each frame boundary, and is proposed for real-time video transmission, such as video conferencing[10]. Although this method improves the network utilization, bandwidth renegotiation is very frequent. The network load will increase because of renegotiation signalling traffic. The other problem with frame period preservation is that the variation of the transmission rate tends to be large. This affects QOS of other multiplexed traffic.
Therefore, video transmission without considering a frame period is superior. In this case, the key is how to avoid buffer underflow and overflow. Next, some existing methods are reviewed.

\subsection{Fixed allocation}

In [6], the minimum bandwidth $R^{*}$ which avoids buffer underflow is allocated. By increasing the STB buffer size and the pre-loading delay, $R^{*}$ can be decreased. The actual transmission rate is controlled to be smaller than or equal to $R^{*}$ to avoid buffer overflow. When an interactive operation changes the frame sequence, $R^{*}$ must be re-calculated. Since this calculation takes time, it is difficult to apply this method to IVOD. Another problem is that the pre-loading delay may be very large. For example, it is $37 \mathrm{~s}$ for Star Wars when the STB memory size is 16 Mbytes.

The Constant-Rate Transmission and Transport (CRTT) method[5] pre-calculates the allocated bandwidth avoiding overflow as well as underflow at the STB buffer. The actual transmission rate is always constant (equal to the allocated bandwidth). Although this method is easy to manage, it is also difficult to apply it to IVOD because of its complex pre-calculation. Moreover, the required STB buffer size and the pre-loading delay are large (in the case of Star Wars, $22.4 M$ bytes memory and $37 s$ pre-loading are required).

\subsection{Dynamic allocation}

If the frame period is preserved in the network, the bandwidth is renegotiated at every frame period. By ignoring the frame period, the renegotiation period can be made much longer. Piecewise Constant Rate Transmission and Transport (PCRTT)[5] is an improved CRTT. By allowing the allocated bandwidth to vary, the required STB buffer size and pre-loading delay can be decreased. The problem is how to determine the scheduling table of the transmission rate. To minimize the impact to other traffic, the transmission rate sequence should be as smooth as possible (i.e. small $\mathrm{CoV}$ of transmission rate). The ideal rate sequence is pureCBR (CRTT realizes this ideal case). The optimal smoothing method[8] tries to find the transmission rate schedule minimizing the $\mathrm{CoV}$ and the peak transmission rate. This method, however, requires a complex pre-calculation. In the case of Star Wars, it takes around $8 s$ when an R4400 ( $150 \mathrm{MHz}$ ) CPU is used. Thus, this scheme can not be used in IVOD.

\section{RCBR based on STB Queue}

All existing methods mentioned above need a complex pre-calculation to obtain the fixed bandwidth or the transmission rate schedule. As a result, it is difficult to apply them in IVOD. Now we propose a new dynamic bandwidth allocation scheme in which no pre-calculation is necessary. 


\subsection{Basic concept}

In order to avoid both underflow and overflow at the STB buffer, the transmission rate and its timing of renegotiation are determined dynamically based on the STB queue length. In this paper, we assume that the backbone and access network is a packet-switched network. Since the routing procedure and the bandwidth allocation can be separated, quick bandwidth renegotiation is possible. For example, bandwidth can be renegotiated using Fast Reservation Protocol (FRP) in the case of ATM[11]. When STB wants to change the allocated bandwidth, it sends a control packet including new bandwidth information to the server. Each intermediate node on the path compares it with the link capacity available. The video server sends back an acknowledgment or reject packet to STB. If at least one intermediate link can not accommodate the requested bandwidth, this FRP is rejected ${ }^{\dagger}$. Therefore, it takes one round-trip time to complete the bandwidth renegotiation. Hereafter, we use the term "FRP" to represent the process of bandwidth renegotiation.

The basic idea of the proposed method, called Dynamic bandwidth Allocation based on Queue length at STB (DAQS), is to increase the bandwidth when a buffer underflow is predicted and to decrease it when a buffer overflow is predicted. The important thing is to determine when the bandwidth should be renegotiated and what the new bandwidth should be.

\subsection{Formulation}

The time axis is divided into discrete units equal to the video frame time. This time index represents the frame number played-back at STB plus the pre-loading time $d$. At the beginning of each frame playback, all information bits in the frame are assumed to be removed from the STB buffer instantaneously. The following random variables and parameters are defined (see Fig. 2).

- $x_{n}$ (bits) : Volume of stored data in STB at $t=n$ just before one frame is removed

- $x_{n}^{+}$(bits) : Volume of stored data in STB at $t=n$ just after one frame is removed

- $y_{n}$ (bits) : Size of the frame played-back at $t=n$

- $r_{n}$ (bits/s) : CBR rate between $t=n$ and $t=n+1$

- $r_{I}($ bits/s) : Initial transmission rate

- F (fps(frame per sec.)) : Reciprocal of frame time length (i.e. frame rate)

- $d$ : Pre-loading time in frame length (first frame is played-back at $t=d$ )

- $B$ (bits) : STB buffer size

- $\omega$ : FRP control delay

- $Y_{\max }$ (bits) : Maximum frame size

- $Y_{\min }$ (bits) : Minimum frame size

- $Y_{a v}$ (bits) : Average frame size

- $V$ : Number of frames in one movie

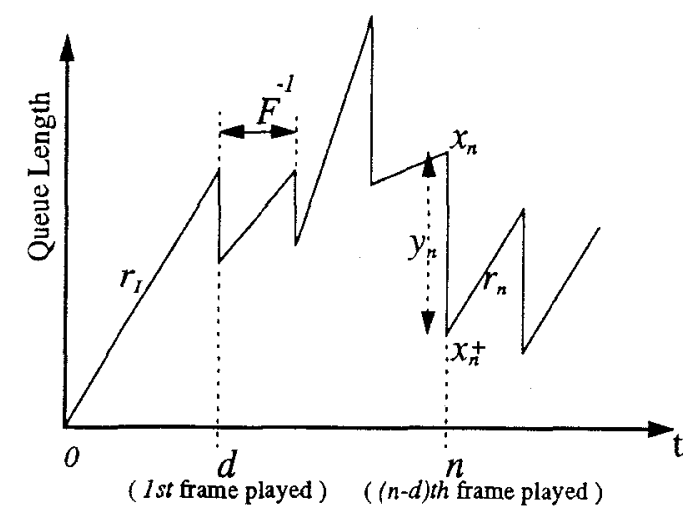

Figure 2: Definition of random variables

If the round-trip time is $T, \omega$ is given by $\omega=\lceil F \cdot T\rceil$ where $\lceil x\rceil$ stands for the minimum integer which is more than or equal to $x$. It is noted that the unit of $\omega$ is the video frame length. Bandwidth renegotiation takes $\omega$ frames, so a change of the transmission rate $r_{n+\omega}$ must be initiated at $t=n$. Therefore, STB must predict the queue length $x_{n+w+1}$ and $x_{n+w+1}^{+}$at $t=n$ to avoid both underflow and overflow at the STB buffer. From Fig. 2,

$$
\left\{\begin{array}{l}
x_{n}=x_{n-1}^{+}+\frac{r_{n-1}}{F} \\
x_{n}^{+}=x_{n}-y_{n}
\end{array}\right.
$$

are obtained. In order to avoid both underflow and overflow at the STB buffer, $x_{n}^{+} \geq 0$ and $x_{n} \leq B$ are required for all $n$. It is assumed that bandwidth is not renegotiated before the playback starts $(t<d)$. So $r_{n}$ can be controlled only when $n \geq d+\omega$. We consider the restrictions for $r_{n}(n \geq d+\omega)$. From (1), the following is derived:

$$
x_{n+1}^{+}=x_{n-\omega}^{+}+\frac{\sum_{i=n-\omega}^{n} r_{i}}{F}-\sum_{j=n-\omega+1}^{n+1} y_{j} .
$$

As $x_{n+1}^{+} \geq 0$ is required, it is necessary that

$$
r_{n} \geq F \sum_{j=n-\omega+1}^{n+1} y_{j}-F x_{n-\omega}^{+}-\sum_{i=n-\omega}^{n-1} r_{i} .
$$

Considering the maximum value of the right side leads to

$$
r_{n} \geq(\omega+1) F Y_{\max }-F x_{n-\omega}^{+}-\sum_{i=n-\omega}^{n-1} r_{i}
$$

In the same way, the following is obtained,

$$
x_{n+1}=x_{n-\omega}^{+}+\frac{\sum_{i=n-\omega}^{n} r_{i}}{F}-\sum_{j=n-\omega+1}^{n} y_{j} .
$$

†For all dynamic bandwidth allocation schemes, there is a possibility the network may reject request for additional bandwidth. In this case, we can just discard some of the frames. 
As $x_{n+1} \leq B$ is required, we have

$$
r_{n} \leq F B+F \sum_{j=n-\omega+1}^{n} y_{j}-F x_{n-\omega}^{+}-\sum_{i=n-\omega}^{n-1} r_{i} .
$$

Considering the minimum value of the right side leads to

$$
r_{n} \leq F B+\omega F Y_{\min }-F x_{n-\omega}^{+}-\sum_{i=n-\omega}^{n-1} r_{i} .
$$

Replacing $n$ with $n+\omega$ in (2) and (3), we obtain the inequality which $r_{n}$ must satisfy for $d \leq n \leq V-\omega$,

$$
(\omega+1) F Y_{\max }-f_{n}\left(x^{+}, \boldsymbol{r}\right) \leq r_{n+\omega} \leq F B+\omega F Y_{\min }-f_{n}\left(x^{+}, \boldsymbol{r}\right)
$$

where

$$
f_{n}\left(x^{+}, r\right) \equiv F x_{n}^{+}+\sum_{i=n}^{n+\omega-1} r_{i} .
$$

For $r_{n}$ to satisfy (4),

$$
B \geq(\omega+1) Y_{\max }-\omega Y_{\min } .
$$

Solving (4) for $x_{n}^{+}$, and setting $r_{n+\omega}=r_{n+\omega-1}$, we have

$$
(\omega+1) Y_{\max }-g_{n}(\boldsymbol{r}) \leq x_{n}^{+} \leq B+\omega Y_{\min }-g_{n}(\boldsymbol{r}) ;
$$

where

$$
g_{n}(r) \equiv \frac{2 r_{n+\omega-1}}{F}+\sum_{i=n}^{n+\omega-2} \frac{r_{i}}{F} .
$$

So long as (6) is satisfied at $t=n$, there is no need to change the transmission rate at $t=n+\omega$, i.e. $r_{n+\omega}=r_{n+\omega-1}$, and FRP at $t=n$ is not necessary. However FRP at $t=n$ is required when (6) is not satisfied. If the bandwidth must be increased, i.e. $x_{n}^{+}<(\omega+1) Y_{\max }-g_{n}(r)$, the new bandwidth at $t=n+\omega$ can be set as

$$
r_{n+\omega}=(\omega+1) F Y_{\max }-f_{n}(x, r),
$$

considering (4). If the bandwidth must be decreased, i.e. $x_{n}^{+}>B+\omega Y_{\min }-g_{n}(r), r_{n+\omega}$ can be set as

$$
r_{n+\omega}=F B+\omega F Y_{\min }-f_{n}(x, r) .
$$

As mentioned before, the transmission rate is kept constant before $t=d+\omega$, i.e.

$$
r_{0}=r_{1}=\cdots=r_{d+\omega-1} \equiv r_{1} .
$$

We must consider the restrictions for $d$ and $r_{I}$ to prevent both overflow and underflow before $t=d+\omega$. From (1), we obtain

$$
\left\{\begin{array}{l}
\max \left(x_{n}\right)=\max \left(x_{n-1}\right)+\frac{r}{F}-Y_{\min }, \\
\min \left(x_{n}^{+}\right)=\min \left(x_{n-1}^{+}\right)+\frac{r_{I}}{F}-Y_{\max }
\end{array}\right.
$$

for $0 \leq n \leq d+\omega$. Assuming that $F Y_{\min } \leq r_{I} \leq F Y_{\max }$, we have

$$
\left\{\begin{array}{l}
\max \left(x_{n}\right) \geq \max \left(x_{n-1}\right), \\
\min \left(x_{n}^{+}\right) \leq \min \left(x_{n-1}^{+}\right)
\end{array}\right.
$$

for $d \leq n \leq d+\omega$. As $y_{n}=0$ when $0 \leq n \leq d-1$, we obtain

$$
\left\{\begin{array}{l}
\max \left(x_{n}\right)>\max \left(x_{n-1}\right), \\
\min \left(x_{n}^{+}\right)>\min \left(x_{n-1}^{+}\right)
\end{array}\right.
$$

for $1 \leq n \leq d-1$. Therefore, we only have to consider $\max \left(x_{d+\omega}\right) \leq B$ and $\min \left(x_{d+\omega}^{+}\right) \geq 0$. From (2) and (3), the following inequalities:

$$
\begin{aligned}
\frac{r_{I}}{F}(d-1)+(\omega+1) \frac{r_{I}}{F}-\omega Y_{\min } & \leq B \\
\frac{r_{I}}{F}(d-1)+(\omega+1)\left(\frac{r_{I}}{F}-Y_{\max }\right) & \geq 0
\end{aligned}
$$

are obtained. Thus the restriction for $d$ and $r_{I}$ is derived as

$$
\left\{\begin{array}{l}
(\omega+1) F Y_{\max } \leq(d+\omega) r_{I} \leq F B+\omega F Y_{\min } \\
F Y_{\min } \leq r_{I} \leq F Y_{\max }
\end{array}\right.
$$

The pre-loading time length $d$ should be as small as possible, so we set $r_{I}$ as the maximum allowed value within $F Y_{\min } \leq$ $r_{I} \leq F Y_{\max }$ at each call set-up time. It means that $r_{I}=$ $\min \left(F Y_{\max }, E\right)$ where $E$ is the minimum available link capacity on the route. Moreover $d$ is derived as

$$
d=\left\lceil\frac{(\omega+1) F Y_{\max }}{r_{I}}\right\rceil-\omega .
$$

For $d$ and $r_{I}$ to exist, the restriction

$$
B \geq(\omega+1) Y_{\max }-\omega Y_{\min }
$$

is necessary. This inequality is the same as (5).

Now, we have determined the bandwidth requirements which avoid both underflow and overflow at the STB buffer. Next, we describe a simple protocol named DAQS-Simplest Protocol (DAQS-SP) to implement these requirements. It is described as follows.

\section{[DAQS-SP]}

Line 1 Transmission starts at $t=0$ with a fixed rate of $r_{I}$. Here, $r_{I}$ satisfies $F Y_{\min } \leq r_{I} \leq F Y_{\max }$ and takes the largest available value.

Line 2 Playback starts at $t=d$. Here, $d=\left\lceil\frac{(\omega+1) F Y_{\max }}{r_{I}}\right\rceil-$ $\omega$.

Line 3 At the instance when one frame is removed from the buffer at $t=n(d \leq n \leq V-\omega)$, the Algorithm Module 1 (AM1) depicted in Fig. 3 is executed.

DAQS-SP guarantees no buffer underflow and overflow so long as FRP is accepted. However, bandwidth is renegotiated only when the STB queue length becomes too short (the buffer underflow is predicted) or too large (the buffer overflow is predicted). Therefore, the allocated bandwidth almost always takes two values: a big one (close to $F Y_{\max }$ ) and a small one (close to $F Y_{\min }$ ). This means the transmission rate will oscillate between two extreme values. In order to decrease the impact to other traffic, the transmission rate should be as smooth as possible. Thus it is a good idea to change the bandwidth gradually. In the next section, a multi-layer concept realizing this modification is proposed. 


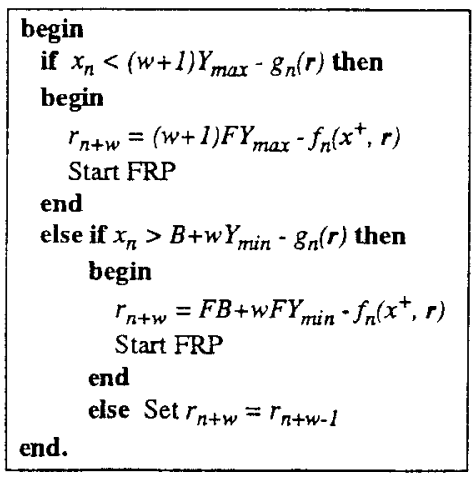

Figure 3: Algorithm module 1

\section{Introduction of Multi-Layer}

The STB buffer is divided into $M(M \geq 3)$ layers. DAQS$\mathrm{SP}$ corresponds to the case of $M=2$. Let $l_{m}(1 \leq m \leq$ $M-1)$ denote the boundary between layer $m-1$ and layer $m$. In layer 0 , the queue length is checked at each frame period based on AM1 to avoid buffer underflow. In layer $M-1$, the queue length is also checked at each frame period based on AM1 to avoid buffer overflow. As a result, the rate is changed dynamically in layer 0 and $M-1$. On the other hand, the volume of video data arriving at STB within a frame period is fixed at $Q_{m}$ for layer $m(1 \leq m \leq M-2)$. If $Q_{m}$ is increased gradually with decreasing $m$, the degree of bandwidth increment and decrement becomes small. So a decrease in the $\mathrm{CoV}$ of the transmission rate is expected. Since the minimum frame size is $Y_{\min }$ and the maximum frame size is $Y_{\max }$, we should set $Q_{m}$ as

$$
Y_{\min }<Q_{M-2}<Q_{M-3}<\cdots<Q_{2}<Q_{1}<Y_{\max }
$$

If the queue length oscillates around a layer boundary, FRP is used in almost every frame. In order to increase the FRP interval, a parameter $\epsilon_{m}(1 \leq m \leq M-1)$ is introduced. If the queue length increases from layer $m-1$ to layer $m$, an FRP changing the transmission rate to $F Q_{m}$ is requested only when the queve length exceeds $l_{m}+\epsilon_{m}$ (see Fig. 4). In the same way, if the queue length decreases from layer $m+1$ to layer $m$, an FRP changing the transmission rate to $F Q_{m}$ is requested only when the queue length falls bellow $l_{m+1}-\epsilon_{m+1}$.

\subsection{Setting $\epsilon_{m}$}

Considering the definition of $Q_{m}$ and $l_{m}$, the allocated bandwidth renegotiation from $F Q_{m-1}$ or $F Q_{m+1}$ to $F Q_{m}$ must be completed when $x_{n}^{+}$is in layer $m$ (i.e. $l_{m} \leq x_{n}^{+} \leq$ $\left.l_{m+1}\right)$. From the worst case ((a) of Fig. 4), the following restriction for $l_{m}(m=1,2, \cdots, M-2)$ is obtained:

$$
l_{m+1}-l_{m} \geq \epsilon_{m}+(\omega+1)\left(Q_{m-1}-Y_{m i n}\right) .
$$

Let $Q_{0}$ denote the maximum transmitted data within one frame in layer 0, i.e. $Y_{\max }$. Moreover, the worst case ((b) of
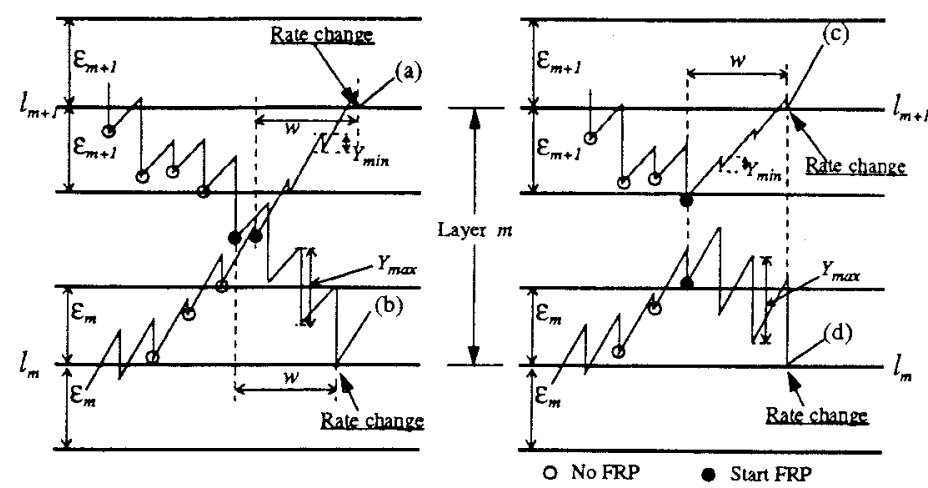

Figure 4: Rate transition in middle layers

Fig. 4) gives the following restriction for $m=1,2, \cdots, M-$ 2:

$$
l_{m+1}-l_{m} \geq \epsilon_{m+1}+(\omega+1)\left(Y_{\max }-Q_{m+1}\right) .
$$

Let $Q_{M-1}$ denote the minimum transmitted data within one frame in layer $M-1$, i.e. $Y_{\min }$. As a result, the restriction for $l_{m}(m=1,2, \cdots, M-2)$ is represented as

$$
\begin{gathered}
l_{m+1} \geq l_{m}+\max \left\{\epsilon_{m}+(\omega+1)\left(Q_{m-1}-Y_{\min }\right),\right. \\
\left.\epsilon_{m+1}+(\omega+1)\left(Y_{\max }-Q_{m+1}\right)\right\} .
\end{gathered}
$$

The worst cases (c) and (d) of Fig. 4 give the restriction $\epsilon_{m} \geq \max \left\{\omega\left(Y_{\max }-Q_{m-1}\right)-1, \omega\left(Q_{m}-Y_{\min }\right)-1\right\}$

for $m=1,2, \cdots, M-1$.

Since $Q_{m}$ is larger when $m$ is smaller, the STB queue should be in as high a layer as possible from the bandwidth consumption point of view. Therefore, the layer interval $l_{m+1}-l_{m}$ for small $m$ should be small. It is indicated by (11) that the layer interval is restricted by $\epsilon_{m}$. Thus $\epsilon_{m}$ should be as small as possible for small $m$. So we introduce a parameter $\kappa(\kappa=0,1, \cdots, M-1)$, and set $\epsilon_{m}=0$ for $1 \leq m \leq \kappa$. Since a large $\epsilon_{m}$ increases the FRP interval, we should make $\epsilon_{m}$ as large as possible for large $m$. Therefore, we introduce a parameter $\alpha(1.0<\alpha)$ and set $\epsilon_{m}$ for $\kappa+2 \leq$ $m \leq M-1$ as

$$
\epsilon_{m}=\epsilon_{\kappa+1} \alpha^{m-\kappa-1},
$$

where $\epsilon_{\kappa+1}$ is set as the maximum value of the right side of (12):

$$
\epsilon_{\kappa+1}=\omega\left(Y_{\max }-Y_{\min }\right)-1 .
$$

Since $\epsilon_{m}(\kappa+2 \leq m \leq M-1)$ is greater than $\epsilon_{\kappa+1}, \epsilon_{m}$ for arbitrary $m$ satisfies (12).

\subsection{Setting $Q_{1}, Q_{M-2}, l_{1}$, and $l_{M-1}$}

Since $Q_{1}$ and $Q_{M-2}$ can be set freely in the region of $Y_{\min }<$ $Q_{M-2}<Q_{1}<Y_{\max }$, let us introduce a parameter $\beta(0<$ $\beta<0.5)$ and set them when $M \geq 4$ as

$$
\begin{cases}Q_{1} & =(1-\beta) Y_{\max }+\beta Y_{\min }, \\ Q_{M-2} & =(1-\beta) Y_{\min }+\beta Y_{\max },\end{cases}
$$


and when $M=3$ as

$$
Q_{1}=Q_{M-2}=(1-2 \beta) Y_{\min }+2 \beta Y_{\max } .
$$

If FRP starts in $x_{n}^{+}=l_{1}-\epsilon_{1}$ and $r_{n+\omega}=F Q_{1}$, the left inequality of (4) becomes

$$
\begin{aligned}
& (\omega+1) F Y_{\max }-F\left(l_{1}-\epsilon_{1}\right)-\sum_{i=n}^{n+\omega-1} r_{i} \leq F Q_{1} . \\
& l_{1} \geq \max \left\{(\omega+1) Y_{\max }-\sum_{i=n}^{n+\omega-1} \frac{r_{i}}{F}-Q_{1}+\epsilon_{1}\right\}
\end{aligned}
$$

is obtained. To minimize the required memory size at STB, $l_{1}$ is set as

$$
l_{1}=(\omega+1)\left(Y_{\max }-Q_{1}\right)+\epsilon_{1} .
$$

We can derive $l_{M-1}$ from $Q_{M-2}$ in the same way:

$$
l_{M-1}=B+\omega Y_{\min }-(\omega+1) Q_{M-2}-\epsilon_{M-1} .
$$

\subsection{Consideration of $l_{m}$}

We have only to consider (11) for determining $l_{m}(m=$ $2,3, \cdots, M-2)$. Let $\mu$ denote the length of layer one $(\mu \equiv$ $\left.l_{2}-l_{1}\right)$. As mentioned before, $\mu$ should be small. From (11), we have

$$
\begin{array}{r}
\mu=\max \left\{\epsilon_{1}+(\omega+1)\left(Y_{\max }-Y_{\min }\right),\right. \\
\left.\epsilon_{2}+(\omega+1)\left(Y_{\max }-Q_{2}\right)\right\} .
\end{array}
$$

To make the layer length $l_{m+1}-l_{m}$ larger as $m$ increases, let us set $l_{m}$ for $3 \leq m \leq M-2$ as

$$
l_{m}=l_{m-1}+\mu \cdot \delta^{m-2}
$$

where $\delta$ is a parameter derived from the buffer size $B$.

There is a minimum required value for $\delta$ to satisfy (11) for arbitrary $m$. Let $\delta_{r e q}^{\left(m^{*}\right)}$ denote the minimum $\delta$ satisfying (11) for $m=m^{*}$. From (11) and (18), $\delta_{r e q}^{\left(m^{*}\right)}$ is derived as

$$
\begin{aligned}
\delta_{r e q}^{\left(m^{*}\right)}= & {\left[\frac { 1 } { \mu } \operatorname { m a x } \left\{\epsilon_{m}+(\omega+1)\left(Q_{m-1}-Y_{\min }\right),\right.\right.} \\
& \left.\left.\epsilon_{m+1}+(\omega+1)\left(Y_{\max }-Q_{m+1}\right)\right\}\right]^{\frac{1}{m}}
\end{aligned}
$$

Moreover let us define $\delta_{\text {req }}$ as

$$
\delta_{r e q} \equiv \max _{2 \leq m^{*} \leq M-2} \delta_{r e q}^{\left(m^{*}\right)} .
$$

The parameter $\delta_{\text {req }}$ represents the minimum required value for parameter $\delta$. The minimum required STB buffer size, $B_{\min }$, is derived from (16) and (17) as follows:

$$
\begin{gathered}
B_{\min }=\mu \cdot \frac{\delta_{r e q}^{M-2}-1}{\delta_{r e q}-1}+(\omega+1)\left(Y_{\max }-Q_{1}+Q_{M-2}\right) \\
-\omega Y_{\min }+\epsilon_{1}+\epsilon_{M-1}
\end{gathered}
$$

Next, let us compute the parameter $\delta$ from a given $B(B \geq$ $B_{\min }$ ). From (16), (17), and (18), we can obtain the following equation for $\delta$ :

$$
\delta^{M-2}-\Gamma \delta+\Gamma-1=0
$$

$$
\begin{gathered}
\Gamma \equiv \mu^{-1}\left\{B+\omega Y_{\min }-(\omega+1)\left(Y_{\max }-Q_{1}+Q_{M-2}\right)\right. \\
\left.-\epsilon_{1}-\epsilon_{M-1}\right\} .
\end{gathered}
$$

\subsection{Setting $Q_{m}$}

We can use any value for $Q_{m}(2 \leq m \leq M-3)$ so long as (10) is satisfied. For example, it is shown that the number of bits per frame obeys the exponential distribution in MPEG[12]. Let $H(y)$ denote the probability distribution function of the number of bits per frame, i.e.

$$
H(y)=1-e^{-\frac{y}{Y_{a v}}},
$$

where $Y_{a v}$ is the average frame size. Now let us determine $H\left(Q_{M-2}\right), H\left(Q_{M-3}\right), \cdots, H\left(Q_{1}\right)$ in the same interval:

$H\left(Q_{m}\right)=H\left(Q_{1}\right)-(m-1)\left(e^{-\frac{Q_{M-3}}{Y_{a v}}}-e^{-\frac{Q_{1}}{Y_{a v}}}\right)(M-3)^{-1}$.

$Q_{m}$ is calculated as

$$
Q_{m}=-Y_{a v} \log \left\{e^{-\frac{Q_{1}}{Y_{a v}}}+\frac{m-1}{M-3}\left(e^{-\frac{Q_{M-2}}{Y_{a v}}}-e^{-\frac{Q_{2}}{Y_{a v}}}\right)\right\} .
$$

$Q_{m}$ can be similarly calculated for other frame size distribution.

\subsection{Initial situation}

It is natural to make $r_{I}$ equal to $F Q_{m}(1 \leq m \leq M-2)$. In this case, $x_{d}^{+}$is in the region:

$$
Q_{m} d-Y_{\max } \leq x_{d}^{+} \leq Q_{m} d-Y_{\min } \text {. }
$$

Since $x_{d}^{+}$should satisfy $l_{m} \leq x_{d}^{+}<l_{m+1}$, we obtain

$$
Q_{m}^{-1}\left(l_{m}+Y_{\max }\right) \leq d<Q_{m}^{-1}\left(l_{m+1}+Y_{\min }\right)
$$

when $r_{I}=F Q_{m}(1 \leq m \leq M-2)$. Since $d$ should be as small as possible, let us set $d$ as

$$
d=\left\lceil Q_{m}^{-1}\left(l_{m}+Y_{\max }\right)\right\rceil
$$

From (11), (16), and (17),

$$
\begin{array}{r}
(d+\omega) r_{I} \geq(\omega+1) F Y_{\max }+F\left\{\sum_{i=1}^{m} \epsilon_{i}+\left(Y_{\max }-Q_{m}\right)\right. \\
\left.+(\omega+1) \sum_{j=1}^{m-1}\left(Y_{\max }-Q_{j}\right)\right\} \\
(d+\omega) r_{I}<F B+\omega F Y_{\min }-F\left\{\sum_{i=m+1}^{M-2} \epsilon_{i}+\left(Q_{m}-Y_{\min }\right)\right. \\
\left.+(\omega+1) \sum_{j=m+1}^{M-2}\left(Q_{j}-Y_{\min }\right)\right\} .
\end{array}
$$


So (9) is satisfied. Among $1 \leq m \leq M-2, m$ is selected for $r_{I}$ as small as possible at each call setup considering the available link capacity.

\subsection{Protocol description}

If the queue length is in layer $m=0$ or $M-1$, AM1 can be used. Now, we describe the advanced protocol. This protocol is named DAQS-Multi-Layer Protocol (DAQS-MLP).

[DAQS-MLP]

Line 1 Transmission starts at $t=0$ with a fixed rate of $r_{I}$. Here, $r_{I}$ satisfies $r_{I}=F Q_{m^{*}}\left(1 \leq m^{*} \leq M-2\right)$ and takes the maximum available value.

Line 2 Playback starts at $t=d\left(d=\left\lceil Q_{m}^{-1} \cdot\left(l_{m} \cdot+Y_{\max }\right)\right\rceil\right)$. The layer indicator $m$ is initialized as $m=m^{*}$.

Line 3 At the instance when one frame is removed from the buffer at $t=n(d \leq n \leq V-\omega)$, the Algorithm Module 2 (AM2) shown in Fig. 5 is executed ( $l_{0} \equiv$ $0, l_{M} \equiv B$ ).

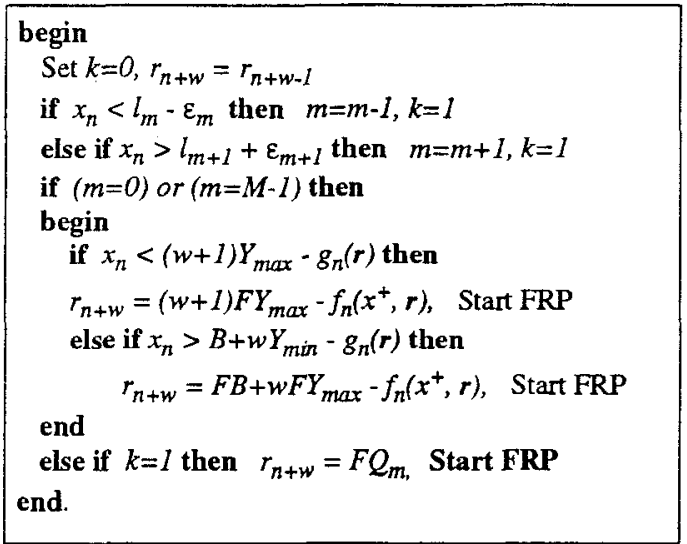

Figure 5: Algorithm module 2

Some interactive operations change the video frame rate. We extend DAQS-SP and DAQS-MLP to interactive operations in [13]. The modified DAQS-SP is called DAQS-SPI (DAQS-SP with Interactive operation), and the modified DAQS-MLP is called DAQS-MLPI (DAQS-MLP with Interactive operation). In this paper, the description of the extension is omitted because of space limitation.

\section{Performance Evaluation}

In this section, we evaluate the performance of the proposed methods. Since no analytical models can adequately represent video traffic, the performance is evaluated by a computer simulator. The distance between video server and STB is assumed to be $50 \mathrm{~km}\left(\omega=\omega_{\max }=\omega_{\min }=1\right)$. Just one $\mathrm{STB}^{\dagger}$ and the video server is considered, and the sim-

\footnotetext{
'Since we assume FRP request will not be rejected, we do not need to simulate multiple STBs.
}

ulation is performed for the whole movie Star Wars [14]. The evaluated value is averaged among 2500 trials.

Twelve successive frames form one segment. The frame rate, $F$, is $24 \mathrm{fps}$, the total number of frames, $V$, is 174128 , the maximum frame size, $Y_{\max }$, is $1.85267 \times 10^{5}$ bits, the minimum frame size, $Y_{\min }$, is 476 bits, and the average frame size, $Y_{a v}$, is $1.55983 \times 10^{4}$ bits.

For the sake of simplicity, only two interactive operations are considered: the slow forward and the fast forward with segment skipping. A fixed slow playback frame rate of $3 f p s$ is used. In the fast playback mode, seven segments are skipped after playingback one segment. Thus this fast playback corresponds to $24 \times 8 \mathrm{fps}$.

The user alternates between normal playback and interactive operation. The duration of normal playback obeys the exponential distribution with mean value $900 s$. The duration of interactive operations also obeys the exponential distribution with mean value $10 \mathrm{~s}$. At each interactive operation, the fast playback and the slow playback are selected with equal probability.

\subsection{Comparison with other methods}

First, the CoV of the transmission rate of DAQS-SPI and DAQS-MLPI are compared with other dynamic bandwidth allocation methods. In particular, one of them preserves the frame period. We call this method BRFP (Bandwidth Renegotiation per Frame Period). In BRFP, the bandwidth is negotiated in every frame period. Besides, the optimal smoothing method[8] (OPT) is also evaluated. It is difficult to apply OPT to the IVOD system because of its complex pre-calculation; however, we can use its performance as an ideal case for the $\mathrm{CoV}$ of the transmission rate. The average pre-loading delay in the optimal smoothing is set to the same value in DAQS $(500 \mathrm{~ms})$. The bandwidth allocation table in OPT is re-calculated when the interactive operation starts or ends. The values of the four parameters in DAQSMLPI are summarized in Table 1 (the influence of these four parameters on performance is evaluated in [13]). $M$ is the number of layers. The layer boundary margin $\epsilon_{m}$ is set to be zero for $1 \leq m \leq \kappa$. The parameter $\alpha$ determines the layer boundary margin $\epsilon_{m}$. The parameter $\beta$ determines $Q_{1}$ and $Q_{M-1}$ through (14) and (15). Here, we assume four STB buffer sizes, $4 M, 8 M, 12 M$, and 16 Mbytes.

Table 1: Values for DAQS-MLPI parameters

\begin{tabular}{|c|c|c|c|c|}
\hline$B$ (Mbytes $)$ & 4 & 8 & 12 & 16 \\
\hline \hline$M$ & 11 & 14 & 13 & 14 \\
\hline$\kappa$ & 1 & 2 & 2 & 2 \\
\hline$\alpha$ & 1.400 & 1.275 & 1.425 & 1.450 \\
\hline$\beta$ & 0.064 & 0.070 & 0.070 & 0.070 \\
\hline
\end{tabular}

In Table 2, the CoV of the transmission rate is summarized. Since the allocated bandwidth takes two extreme values in DAQS-SPI, the $\mathrm{CoV}$ of the transmission rate is 
Table 2: Comparison of the $\mathrm{CoV}$ of the transmission rate

\begin{tabular}{|c|c|c|c|c|}
\hline$B$ (Mbytes $)$ & 4 & 8 & 12 & 16 \\
\hline \hline BRFP & 1.1652 & 1.1652 & 1.1652 & 1.1652 \\
\hline DAQS-SPI & 1.0721 & 1.1889 & 1.2849 & 1.3605 \\
\hline DAQS-MLPI & 0.2471 & 0.2242 & 0.2126 & 0.2094 \\
\hline OPT & 0.1575 & 0.1385 & 0.1336 & 0.1252 \\
\hline
\end{tabular}

very large compared with DAQS-MLPI and the optimal value. It should be noted that DAQS-MLPI shows a good performance close to the optimal. For example, the $\mathrm{CoV}$ in DAQS-MLPI is 0.2471 and the optimal value is 0.1575 when $B=4.0$ Mbytes.

Table 3: Comparison of bandwidth utilization

\begin{tabular}{|c|c|c|c|c|}
\hline$B$ (Mbytes $)$ & 4 & 8 & 12 & 16 \\
\hline \hline PRA & 0.0842 & 0.0842 & 0.0842 & 0.0842 \\
\hline MBA & 0.7454 & 0.8161 & 0.8981 & 1.0 \\
\hline DAQS-SPI & 0.9680 & 0.9459 & 0.9184 & 0.8949 \\
\hline DAQS-MLPI & 0.9706 & 0.9439 & 0.9317 & 0.9189 \\
\hline OPT & 0.9785 & 0.9598 & 0.9449 & 0.9357 \\
\hline BRFP & 1.0 & 1.0 & 1.0 & 1.0 \\
\hline
\end{tabular}

In Table 3, the bandwidth utilization in DAQS-SPI and DAQS-MLPI are compared with other bandwidth allocation methods in VOD. Since the required STB memory size in CRTT is much larger than assumed memory size, the minimum bandwidth allocation method[6] called MBA (Minimum Bandwidth Allocation) and OPT are evaluated. We also show the case of BRFP and the Peak Rate Allocation (PRA) method. Generally speaking, increase in the STB memory makes the allocated bandwidth larger. So the bandwidth utilization in DAQS and OPT degrades as the memory size increases. Although MBA may show good performance when the STB memory size is large, huge preloading may be required (it depends on the video source and the memory size. In the case of 16 Mbytes STB memory, the pre-loading delay is $37 s$ ). On the other hand, DAQS-SPI and DAQS-MLPI require short pre-loading delay $(500 \mathrm{~ms})$.

From Tables 2 and 3 , it is concluded that DAQS-MLPI has excellent characteristics compared with other bandwidth allocation methods in VOD.

\section{Conclusion}

A new bandwidth allocation method for the IVOD system with constant quality is proposed. This method, called DAQS, dynamically renegotiates the allocated bandwidth based on the STB queue length to avoid both buffer underflow and overflow. No complex pre-calculation is nec- essary, so this method can be applied to IVOD. Moreover, the multi-layer concept is introduced to decrease the $\mathrm{CoV}$ of the transmission rate.

Through a simulation model, it is shown that the CoV of the transmission rate in DAQS-MLPI is close to the optimal and the bandwidth utilization is close to unity. So we conclude that DAQS-MLPI is an excellent bandwidth allocation method suited for IVOD. It is noted that this method can be used for any stored video service with interactivity.

\section{References}

[1] Y. Chang et al. "An Open-Systems Approach to Video on Demand". IEEE Communication Magazine, pages 68-80, 51994.

[2] D. Deloddere, W. Verbiest, and H. Verhille. "Interactive Video On Demand". IEEE Communication Magazine, pages 82-88, 51994.

[3] D. Reininger and D. Raychaudhuri. "Bit-Rate Characteristics of a VBR MPEG Video Encoder for ATM Networks". In IEEE ICC'93, pages 517-521, 1993.

[4] C. Huang, M. Devetsikiotis, I. Lambadaris, and A.R. Kaye. "Modeling and Simulation of Self-Similar Variable Bit Rate Compressed Video: A Unified Approach". In ACM SIGCOMM'95, pages 114-125, 1995.

[5] J.M. McManus and K.W. Ross. "Video-on-Demand Over ATM: Constant-Rate Transmission and Transport". IEEE Journal on Selected Areas in Communications, 14(6):10871098, 81996.

[6] J. Lauderdale and D.H.K. Tsang. "Using the Minimum Reservation Rate for Transmission of Pre-Encoded MPEG VBR Video Using CBR Service". IEICE Trans. Communications, pages 1023-1029, 81996.

[7] M. Grossglauser, S. Keshav, and D. Tse. "RCBR: A Simple and Efficient Service for Multiple Time-Scale Traffic". In ACM SIGCOMM'95, pages 114-125, 1995.

[8] J.D. Salehi, Z. Zhang, J.F. Kurose, and D. Towsley. "Supporting Stored Video: Reducing Rate Variability and Endto-End Resource Requirements through Optimal Smoothing". In ACM SIGMETRICS'96, 51996.

[9] H. Zhang and E. Knightly. "A New Approach to Support Delay-Sensitive VBR Video in Packet-Switched Networks". In 5 th Workshop on Networking and Operating System Support for Digital Audio and Video, pages 275-286, 41995.

[10] S. El-Henaoui, R. Coelho, and S. Tohme. "A Bandwidth Allocation Protocol for MPEG VBR Traffic in ATM Networks". In IEEE INFORCOM'96, pages 1100-1107, 31996.

[11] H. Shimonishi, T. Takine, M. Murata, and H. Miyahara. "Performance Analysis of Fast Reservation Protocol with Generalized Bandwidth Reservation Method". In IEEE INFORCOM'96, pages 758-767, 31996.

[12] C.S. Freedman and D.J. DeWitt. "The SPIFFI Scalable Video-on-demand System". In ACM SIGMOD'95, pages 352-363, 1995.

[13] N. Kamiyama and V.O.K. Li. "Dynamic Bandwidth Allocation based on Queue Length of STB in IVOD System". submitted to IEEE/ACM Trans. on Networking.

[14] M.W. Garrett and W. Willinger. "Analysis, Modeling and Generation of Self-Similar VBR Video Traffic". In $A C M$ SIGCOMM'94, pages 269-280, 1994. 